\title{
TUTORIAL CONVIDADO
}

\section{SIMULAÇÃO-OTIMIZAÇÃO: PORQUE E COMO COMBINÁ-LAS? ${ }^{1}$}

\author{
Gonçalo Figueira $^{a^{*}}$ \\ ${ }^{a}$ INESC TEC, Faculdade de Engenharia \\ Universidade do Porto, Porto, Portugal
}

\section{Introdução}

Simulação e otimização são duas das principais áreas da Pesquisa Operacional, sendo também conceitos amplos e que por vezes adquirem significados distintos em diferentes contextos. Simulação pode ser genericamente definida como "a imitação do funcionamento de um processo ou sistema real" (Banks, 1999). Ora, nesta definição cabe um conjunto muito alargado de técnicas, da simulação estatística (ou Monte Carlo) à simulação dinâmica, da discreta à contínua, da baseada em agentes aos jogos de computador (em particular os chamados "jogos sérios", como por exemplo os simuladores de aviões). O contexto do uso da simulação pode também variar, desde o apoio no desenho de sistemas de engenharia ao treino de profissionais para a execução de uma certa tarefa, ou mesmo o estudo da interação de agentes económicos num dado mercado. A otimização, por seu lado, está muito associada ao conceito de "programação matemática" e pode apoiar precisamente nos mesmos problemas.

No contexto específico da PO, i.e., na resolução de problemas de decisão, a simulação e a otimização são tipicamente vistas como formas alternativas de abordar o problema. Uma vez que a simulação é capaz de incorporar um elevado nível de detalhe nos modelos, sem recorrer a grande sofisticação matemática, é muitas vezes usada em problemas onde, pelo seu nível de complexidade, a tradução num modelo analítico seria inviável. Um exemplo típico é a definição de layouts, que frequentemente exige modelar sistemas complexos de filas de espera com múltiplos fluxos e regras de despacho. Por outro lado, a necessidade de usar otimização é evidente em problemas que envolvem um alargado conjunto de decisões, quer contínuas, quer combinatórias, como o caso dos problemas de corte e empacotamento, o roteamento de veículos, ou o planejamento e escalonamento da produção. Sendo a simulação e a otimização duas abordagens tão distintas, duas grandes questões podem ser colocadas: porque e como combiná-las? Este artigo procura dar algumas respostas, fazendo uma breve introdução à vasta literatura existente.

\section{Porque Combinar Simulação e Otimização?}

Esta questão pode ser vista de duas perspectivas: porque combinar simulação com otimização; ou porque combinar otimização com simulação. Relativamente à primeira, a verdade é que a simulação tem um carácter descritivo ou preditivo, enquanto a otimização tem uma natureza prescritiva. Assim, a simulação carece sempre de um procedimento, ainda que

*Autor para correspondência. E-mail: goncalo.figueira@fe.up.pt

DOI: 10.4322/PODes.2019.001

${ }^{1}$ Este texto foi originalmente publicado no Boletim APDIO, 58:9-11 (2018),

http://apdio.pt/documents/10180/16684/AF_Boletim\%20APDIO_58_web.pdf. A Revista PODes

agradece ao autor e aos Editores do Boletim da APDIO pela oportunidade de republicar este artigo. 
manual, de definição das várias alternativas que devem ser testadas e de onde surgirá a escolha final. O considerável aumento de poder computacional tem permitido abordar problemas cada vez mais complexos e, assim, alargar o âmbito das decisões. Neste contexto, a necessidade de ter um procedimento automático torna-se cada vez mais relevante. Por outro lado, para além do espaço de solução, a otimização de problemas estocásticos pode envolver ainda um outro espaço de pesquisa, o espaço de probabilidade. Ou seja, duas soluções terão de ser simuladas várias vezes para se perceber qual delas responde melhor ao objetivo pretendido. Quantas réplicas realizar, que cenários testar e como alternar entre os espaços de solução e probabilidade são questões que devem ser respondidas e que beneficiam de um procedimento estruturado e adaptativo.

Na segunda perspectiva, o objetivo é usar a simulação para ajudar a otimização, endereçando os principais desafios dos modelos analíticos: variáveis categóricas, relações não lineares, incerteza ou mesmo processos complexos que não podem ser modelados por expressões puramente analíticas. A incerteza, por exemplo, é um elemento vastamente estudado, existindo uma variedade de técnicas de otimização para a abordar, incluindo a programação estocástica, a otimização robusta e a lógica difusa. Porém, na maioria dos casos, ou os modelos são substancialmente mais complexos comparativamente com os seus correspondentes determinísticos, ou apresentam um elevado grau de abstração que pode colocar em causa a sua adesão à realidade, ou ambas as coisas. Isto é particularmente evidente quando se procura modelar por exemplo distúrbios, i.e., episódios com início e fim determinados que afetam consideravelmente o desempenho do sistema. Ora, a simulação por eventos discretos lida muito facilmente com estes fenómenos, enquanto os modelos analíticos são obrigados a recorrer a aproximações arrojadas. Muitos decisores contornam esta questão introduzindo folgas nos seus modelos analíticos, que visam acomodar este tipo de incerteza. Contudo, a determinação dessas folgas é feita de forma arbitrária e, por isso, poderá conduzir a uma solução de qualidade medíocre.

Estas duas perspectivas levaram pesquisadores de ambas as áreas a procurar estender os seus métodos com técnicas da outra área. Abordagens baseadas em simulação começaram a envolver a otimização dos parâmetros de entrada da simulação, i.e., dos chamados "cenários de decisão" (otimização da simulação). Abordagens baseadas em otimização começaram a usar simulação para o cálculo de parâmetros ou a amostragem de cenários para modelos de programação estocástica (simulação para a otimização). No entanto, esta dicotomia está gradualmente a desaparecer, uma vez que alguns métodos combinam simulação e otimização de uma forma mais equilibrada. Todos os métodos que combinam de alguma forma simulação e otimização podem ser genericamente designados por "métodos híbridos de simulaçãootimização", ou simplesmente por "simulação-otimização" (S-O) (Figueira e Almada-Lobo, 2014).

\section{Como Combinar Simulação e Otimização?}

Existem formas muito distintas de combinar simulação e otimização, sendo que não existe uma que se revele superior às outras para um conjunto de problemas suficientemente diverso. A forma ideal depende portanto das características do problema. Um dos principais aspetos nestas técnicas é, como seria de esperar, o compromisso entre exatidão do modelo/método e esforço computacional. Dado que a simulação consegue representar a realidade com mais detalhe e precisão, a sua utilização na avaliação de soluções deverá permitir realizar uma pesquisa consistente, mas poderá requerer um tempo computacional proibitivo, visto que cada solução terá de ser simulada (múltiplas vezes no caso de problemas estocásticos). Assim, a questão fundamental a responder é: até que ponto os modelos analíticos nos são úteis e quando precisamos verdadeiramente da simulação? Se não for possível construir a priori um modelo analítico razoável, a simulação terá de facto de ser usada para avaliar soluções. No caso de o problema apresentar uma estrutura onde os modelos analíticos trazem alguma vantagem, esse deverá então ser o ponto de partida e a simulação ser usada para os complementar ou melhorar, nos chamados "modelos híbridos" ou "modelagem híbrida". No restante artigo, estas 
duas principais abordagens são exploradas, bem como uma terceira, que pode ser vista como pertencendo a uma e a outra, e que tem que ver com o aprendizado de regras num ambiente de simulação. Uma taxonomia completa dos métodos de S-O pode ser encontrada em (Figueira e Almada-Lobo, 2014). A Figura 1 exibe de uma forma resumida as várias categorias de métodos de S-O.

Figura 1: Principais dimensões na classificação dos métodos de S-O: para cada propósito da simulação, é indicada a estrutura típica, embora outras sejam possíveis.

\begin{tabular}{|c|c|c|c|c|c|}
\hline \multirow{3}{*}{$\begin{array}{l}\text { Propósito da } \\
\text { Simulação }\end{array}$} & \multicolumn{2}{|c|}{ Simulação para Avaliar Soluções (SAS) } & \multicolumn{2}{|c|}{ Modelos/Modelagem Híbrida (MH) } & \multirow{2}{*}{$\begin{array}{l}\text { Aprendizado de Regras } \\
\text { (AR) }\end{array}$} \\
\hline & Função Avaliadora & $\begin{array}{l}\text { Construção de } \\
\text { Meta-modelo }\end{array}$ & $\begin{array}{l}\text { Melhoramento do } \\
\text { Modelo Analítico }\end{array}$ & Geração da Solução & \\
\hline & & & & & \\
\hline \multirow{2}{*}{$\begin{array}{l}\text { Estrutura } \\
\text { Típica }\end{array}$} & Otimização & Simulação & Simulação & Simulação & Otimização \\
\hline & Simulação & & & Otimizaç̃ão & Otimização \\
\hline
\end{tabular}

Fonte: Adaptado de Figueira e Almada-Lobo (2014).

\subsection{Simulação para Avaliar Soluções (SAS)}

Quando o espaço de solução é muito reduzido, as soluções podem ser todas simuladas e avaliadas de forma a selecionar a melhor. Contudo, tal como referido anteriormente, em problemas estocásticos o espaço de probabilidade deverá também ser explorado. Os métodos de seleção estatística (Chen et al., 2015) visam justamente determinar o número de réplicas da simulação, ou a sua duração, que permite selecionar uma solução em detrimento de outra, com um certo nível de confiança. Este número poderá não ser o mesmo para todas as soluções, já que uma solução de baixa qualidade rapidamente conseguirá ser rejeitada. Para além do número de réplicas, a definição dos próprios cenários a testar pode ser alvo de análise. As técnicas de redução de variância (Homem-de-Mello e Bayraksan, 2015) abordam precisamente este tema, conseguindo reduzir a variância dos resultados para o mesmo número de réplicas, que é o mesmo que dizer que para o mesmo nível de confiança permitem reduzir o número de réplicas e, assim, o esforço computacional.

Explorar amplos espaços de solução exige, por outro lado, verdadeiros métodos de otimização. As heurísticas e as meta-heurísticas são candidatos interessantes, pela sua flexibilidade em se adaptarem a diferentes problemas e, neste caso em particular, facilmente incluírem um modelo de simulação na sua função avaliadora. A sua popularidade é especialmente elevada em problemas de otimização combinatória (Juan et al., 2015). Em espaços contínuos, a pesquisa baseada em gradientes surge com frequência na literatura de S-O (Fu, 2015), uma vez que a última pode ser vista como uma extensão da programação não linear.

Não obstante, todos estes métodos requerem o uso da simulação para cada solução que é visitada, resultando num elevado tempo computacional, que pode ser inviável em muitos casos. Uma forma de contornar este problema consiste em aproximar um meta-modelo à "paisagem" de soluções (Dellino et al., 2015). Ou seja, em vez de iterar por diferentes soluções e correr simulações para cada, um conjunto limitado de pontos é selecionado, simulado e o resultado usado para aproximar um modelo analítico, geralmente não linear, no qual uma pesquisa rápida pode ter lugar, i.e., sem recorrer a mais simulações. 


\subsection{Modelos/Modelagem Híbrida (MH)}

Os métodos anteriormente descritos, mesmo os baseados em meta-modelos, assumem que não é possível construir a priori um modelo analítico que descreva razoavelmente bem o problema e que tenha uma complexidade tratável. Aqui, o termo "modelo analítico" refere-se não só a modelos de programação matemática, mas mais genericamente a qualquer modelo ou método que use exclusivamente expressões analíticas para definir o problema, como é o caso das meta-heurísticas. Se tal modelo analítico existir, então deverá ser usado conjuntamente com a simulação. Shanthikumar e Sargent (1983) distinguem entre "modelos híbridos", onde os modelos analítico e de simulação são combinados num único, e "modelagem híbrida", que se refere a usar dois modelos que individualmente conseguem gerar uma solução completa, mas que trocam informação entre si na geração da solução final. Uma questão crítica neste caso é garantir a coerência entre os diferentes níveis de granularidade que caracterizam os modelos analítico e de simulação.

Uma outra distinção, talvez até mais relevante, prende-se com o papel que a simulação assume neste método híbrido. Em certos problemas, o resultado da simulação pode não ser essencial para a escolha da solução. Nesses casos, os modelos analíticos podem ser formulados e resolvidos, e as suas soluções simuladas, por forma a determinar todas as variáveis de interesse. O propósito da simulação aqui não é o de verificar a vantagem de uma solução relativamente a outra, mas simplesmente ser parte da geração da solução. Todavia, quando a escolha de alguma decisão depende do resultado da simulação, que será provavelmente a maioria dos casos, ou o método anterior é combinado com um SAS, e a solução é iterativamente alterada até ser satisfatória, ou então a simulação terá que de alguma forma modificar o modelo analítico para que novas e melhores soluções sejam geradas.

Existe uma vasta literatura debruçada sobre este tema, que considera diferentes formas de modificar os modelos analíticos utilizando o resultado da simulação. Tipicamente isto é feito refinando parâmetros críticos, como capacidades, tempos ou custos. Na maioria dos casos, esse refinamento visa criar alguma folga para acomodar incerteza. Porém, pode também ser usado para lidar com não-linearidades, particularmente funções definidas por partes, onde a simulação aponta em cada momento a parte a ser considerada no modelo analítico (Almeder et al., 2009).

\subsection{Aprendizado de Regras (AR)}

O tão badalado aprendizado de máquina (machine learning), agora na ribalta em virtude da crescente capitalização do seu potencial, tem uma forte relação com a S-O. Genericamente, os métodos de aprendizado de máquina têm por objetivo treinar algoritmos na execução de determinadas tarefas, como classificação ou regressão. No âmbito da PO, o aprendizado de máquina foca-se normalmente em algoritmos que resolvem problemas de otimização. Estes algoritmos consistem em regras ou políticas simples, que podem ser executadas num curto espaço de tempo. São por isso métodos ideais para abordar problemas dinâmicos, altamente estocásticos, que curiosamente são os mesmos onde a simulação é necessária.

Aqui, a interligação entre simulação e otimização é grande. A regra de decisão é corrida dinamicamente ao longo da simulação. No entanto, este "modelo híbrido" representa apenas a avaliação da eficácia de uma regra em particular. A regra em si, a sua forma ou os seus parâmetros, requer uma meta-otimização. Esta combinação de S-O pode ser portanto vista como uma mistura das duas grandes abordagens acima descritas: um modelo híbrido (MH) que é usado para avaliar soluções (SAS), sendo que uma solução neste caso representa uma regra.

Por exemplo, na gestão de inventário, a regra a otimizar pode corresponder à forma como se determina a política de reaprovisionamento, constituída por um ou mais parâmetros. Ou seja, enquanto um método SAS utiliza a otimização para determinar diretamente o valor dos parâmetros (por exemplo, o ponto e a quantidade de encomenda) e a simulação para a testar, o aprendizado de regras consiste em determinar um algoritmo, que pode ser uma simples expressão matemática, e que irá calcular os parâmetros da política. Para tal, aplicam-se métodos como redes neuronais, árvores de decisão ou programação genética (Nguyen et al., 2017). 


\section{Considerações Finais}

São vários os problemas que podem beneficiar destes tipos de técnicas, incluindo o roteamento de veículos, o escalonamento da produção, e a movimentação ou alocação de produtos no chão de fábrica. Na nova era da Indústria 4.0, onde por um lado os sistemas de produção são cada vez mais complexos, mas por outro começa a existir mais informação em tempo real sobre o estado dos recursos, há uma tendência crescente para métodos de decisão descentralizados, ágeis e rápidos. A S-O deverá por isso ter um papel fundamental na resolução destes problemas.

Agradecimentos. O autor deste trabalho agradece aos seus co-autores, em particular a Bernardo Almada Lobo e Christian Almeder, pelas várias contribuições em diversos trabalhos nesta área, que ajudaram a ganhar uma perspectiva abrangente sobre o tema e, assim, a estruturar o conteúdo deste tutorial. $\mathrm{O}$ autor agradece também à Fundação para a Ciência e Tecnologia (FCT) e ao INESC TEC pelo apoio financeiro e institucional, e ainda à Editora-Chefe o convite e a ajuda na adaptação deste tutorial.

\section{Referências}

Almeder, C., Preusser, M. e Hartl, R. F. Simulation and optimization of supply chains: alternative or complementary approaches? OR Spectrum, v. 31, n. 1, p. 95-119, 2009.

Banks, J. Introduction to simulation. In Winter Simulation Conference Proceedings, v. 1, p. 7 13, 1999.

Chen, C.-H., Chick, S. E., Lee, L. H. e Pujowidianto, N. A. Ranking and selection: efficient simulation budget allocation. In: Fu, M. C. (ed.) Handbook of Simulation Optimization, v. 216, p. 45-80. New York, NY: Springer, 2015.

Dellino, G., Kleijnen, J. P. C. e Meloni, C. Metamodel-based robust simulation-optimization: An overview. In: Dellino, G., Meloni, C. (eds.) Uncertainty Management in SimulationOptimization of Complex Systems, v. 59, p. 27-54. Boston, MA: Springer, 2015.

Figueira, G. e Almada-Lobo, B. Hybrid simulation-optimization methods: A taxonomy and discussion. Simulation Modelling Practice and Theory, v. 46, p. 118-134, 2014.

Fu, M. C. Stochastic gradient estimation. In: Fu, M. C. (ed.) Handbook of Simulation Optimization, v. 216, p. 105-147. New York, NY: Springer, 2015.

Homem-de-Mello, T. e Bayraksan, G. Stochastic constraints and variance reduction techniques. In: Fu, M. C. (ed.) Handbook of Simulation Optimization, v. 216, p. 245-276. New York, NY: Springer, 2015.

Juan, A. A., Faulin, J., Grasman, S. E., Rabe, M. e Figueira, G. A review of simheuristics: Extending metaheuristics to deal with stochastic combinatorial optimization problems. Operations Research Perspectives, v. 2, p. 62-72, 2015.

Nguyen, S., Mei, Y. e Zhang, M. Genetic programming for production scheduling: a survey with a unified framework. Complex \& Intelligent Systems, v. 3, n. 1, p. 41-66, 2017.

Shanthikumar, J. G. e Sargent, R. G. A unifying view of hybrid simulation/analytic models and modeling. Operations Research, v. 31, n. 6, p.1030-1052, 1983. 